\title{
Self-assembly of cholesterol tethered within hydrogel networks
}

Kristin Engberg ${ }^{a}$, Dale J. Waters ${ }^{a}$, Shira Kelmanovich ${ }^{a}$, Rachel Parke-Houben ${ }^{a}$, Laura

Hartmann ${ }^{a}$, Michael F. Toney ${ }^{b}$, and Curtis W. Frank ${ }^{a *}$

${ }^{\mathrm{a}}$ Department of Chemical Engineering, Stanford University, 443 Via Ortega, Shriram Center,

Stanford, CA 94305-4125, USA

${ }^{\mathrm{b}}$ Stanford Synchrotron Radiation Lightsource, Menlo Park, CA 94025-7015, USA

*Corresponding author; Tel: +1 650723 4573; fax: +1 650723 9780;

Email: curt.frank@stanford.edu

(C) 2016. This manuscript version is made available under the Elsevier user license http://www.elsevier.com/open-access/userlicense/1.0/ 


\begin{abstract}
Cholesterol self-assembles into weakly ordered aggregates when tethered to a crosslinked hydrogel network of poly(ethylene glycol) (PEG). PEG-diacrylate and cholesterol-PEGacrylamide (PEG-chol) were co-polymerized in organic solvent and transferred to water for equilibrium swelling. Small-angle x-ray scattering revealed self-assembled cholesterol structures not present during network synthesis. At lower ratios of PEG-tethered cholesterol to PEG ( $<12 \%$ cholesterol based on total solid content), cholesterol aggregates into the dense, weakly ordered crosslink junctions of the PEG network. The hydrogel networks exhibited classic affine behavior during compressive mechanical testing, and cholesterol aggregation enhanced the elastic modulus. At high PEG-chol to PEG ratios (12-20\% cholesterol based on total solid content), cholesterol self-assembles into domains with lamellar-like meso-ordering. The structural transition causes network deswelling and significantly reduces material brittleness upon deformation.
\end{abstract}

\title{
Keywords
}

Hydrogel network; Self-assembly; SAXS

\section{Introduction}

Hydrogel materials are notably responsive to changes in their chemical composition and method of network formation. Consequently, they can be designed to exhibit a variety of properties, such as high absorbability[1-5], desired molecular transport[6-8], injectability[9-12], biodegradability[12-14], high strength[15-18], extensibility[2, 19-21], and/or toughness[2, 1825]. Small-angle $\mathrm{x}$-ray and neutron scattering (SAXS/SANS) have proven to be useful tools to probe nanoscale structural features of hydrogel networks in order to gain insight into the macroscopic properties and their dependence on composition[26]. For example, our previous 
work established that photopolymerized, end-linked poly(ethylene glycol) (PEG) networks produce a scattering peak that corresponds to the distance between weakly ordered crosslinks. The crosslink junctions were determined to be dense, hydrophobic scattering centers with high estimated functionality (over 80 PEG chains per junction), and they contribute significantly to enhance the mechanical strength of the PEG hydrogel network[27]. By comparison, end-linked "tetra-PEG" networks with completely uniform, homogenous structures of exactly four chains per crosslink show no heterogeneous scattering features[28, 29]. Although such networks do not have the high initial elastic moduli of the photopolymerized PEG hydrogels[25, 27, 28], their uniform structure apparently causes them to be substantially less brittle, and they instead demonstrate enhanced toughness and fracture strength[25].

Clearly, chemical composition strongly impacts the structure/property relationships of hydrogel networks, but the processing of the network after synthesis can be equally important. In the case of temperature-sensitive polymers, thermal processing can produce physical crosslinks or act as a method of drug delivery $[9,12,30]$. When photopolymerized PEG is interpenetrated by a network of $\mathrm{pH}$-sensitive poly(acrylic acid) (PAA), the resulting double network exhibits an exceptional increase in elastic modulus when the PAA is ionized compared to when it is protonated or incorporated as a copolymer with PEG[15, 31]. In such instances, the swelling behavior of the polymer chains is strongly influenced by changes to the network environment. The structural transition caused by altering the hydrogel environment further influences the material properties.

In the current study, the effects of both composition and processing were explored as cholesterol was incorporated into end-linked PEG networks using a tethering PEG-linker to connect the cholesterol groups to a network junction. PEG-co-(PEG-chol) networks ranging 
from 0 to $100 \%$ PEG-chol were photopolymerized in chloroform and swollen to equilibrium in water. As a hydrophobic mesogen, cholesterol easily dissolves in organic solvent for network synthesis, yet it has the capability to stack or self-assemble once forced into aqueous conditions. Tethering cholesterol to the PEG network junctions generates a homogeneous distribution throughout the hydrogel and permits greater mobility for molecular rearrangement compared to direct incorporation into the polymer backbone. However, attachment via a polymer linker restricts movement to a local region, which may limit self-assembly within the network. This study uses SAXS to analyze the structural behavior of the PEG-co-(PEG-chol) networks as a result of solvent processing and cholesterol concentration. The translation of the nanostructure to the swelling and mechanical properties of the hydrogels is also examined.

\section{Experimental}

\section{Chemicals}

Diol-terminated PEG ( $M_{n} 4600$ and $3400 \mathrm{~g} / \mathrm{mol}$ ), bis(3-aminopropyl)-terminated PEG $\left(M_{n} 1500 \mathrm{~g} / \mathrm{mol}\right)$, PEG methyl ether acrylate $\left(M_{n} 2000 \mathrm{~g} / \mathrm{mol}\right)$, triethylamine, acryloyl chloride, cholesteryl chloroformate, mesyl chloride, ammonia, 2-hydroxy-2-methylpropiophenone and triethylene glycol dimethacrylate were purchased from Sigma-Aldrich (St. Louis, MO) and used as received. Anhydrous tetrahydrofuran, diethyl ether, dichloromethane and chloroform were purchased from Fisher Scientific (Pittsburgh, PA). Ethanol was purchased from Rossville Gold Shield (Hayward, CA).

\section{PEG-diacrylate and PEG-diamine synthesis}

PEG-diacrylate $\left(M_{n} 4600 \mathrm{~g} / \mathrm{mol}\right)$ and PEG-diamine $\left(M_{n} 3400 \mathrm{~g} / \mathrm{mol}\right)$ were synthesized following a protocol similar to that described by Elbert and Hubbell[32]. Briefly, diolterminated PEG dissolved in anhydrous tetrahydrofuran was reacted with a 2.5 molar excess of 
acryloyl chloride (for PEG-diacrylate) or a 3 molar excess of mesyl chloride (for PEG-diamine) and triethylamine base catalyst overnight at room temperature under nitrogen. The PEGdiacrylate and PEG-dimesylate products were then purified by filtration and precipitation in diethyl ether. The PEG-dimesylate was added to an aqueous ammonia solution, and the reaction was stirred for a week to produce PEG-diamine. Further purification of the PEG-diacrylate (Figure 1a) and PEG-diamine products was performed using liquid-liquid extraction in dichloromethane and precipitation in diethyl ether. ${ }^{1} \mathrm{H}$ NMR analysis confirmed full end-group functionalization, as described previously[27].

(a)

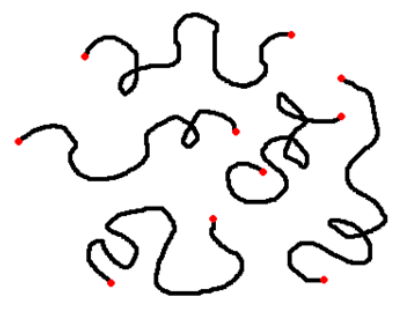

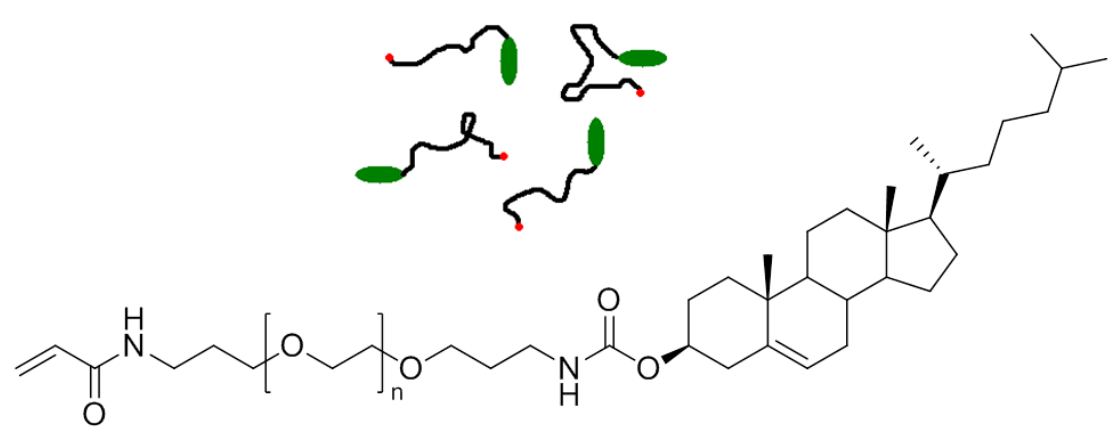

Figure 1. (a) PEG-diacrylate chemical structure ( $\mathrm{n} \approx 104$ for $M_{n} 4600 \mathrm{~g} / \mathrm{mol}$ ) and cartoon graphic where black lines represent PEG chains and red circles represent hydrophobic acrylate end-groups. (b) PEG-chol chemical structure ( $\mathrm{n} \approx 34$ for PEG $\left.M_{n} 1500 \mathrm{~g} / \mathrm{mol}\right)$ and cartoon graphic where black lines represent PEG chains, red circles represent hydrophobic acrylamide end-groups and green ovals represent hydrophobic cholesterol molecules.

\section{Cholesterol-PEG-acrylamide (PEG-chol) synthesis}

3 grams of amine-terminated PEG ( $2 \mathrm{mmol} M_{n} 1500 \mathrm{~g} / \mathrm{mol}, \sim 34$ repeating units (r.u.); 0.9 mmol $M_{n} 3400 \mathrm{~g} / \mathrm{mol}, \sim 77$ r.u.) were dissolved in $\sim 150 \mathrm{~mL}$ of dichloromethane, and 2 molar equivalents of distilled triethylamine per PEG chain were added. A solution of 1 molar equivalent cholesteryl chloroformate per PEG chain in $\sim 20 \mathrm{~mL}$ dichloromethane was slowly dripped into the reaction flask (about 1 drop every 5-10 seconds) to promote the addition of a cholesterol group to only one end of each PEG chain. After stirring overnight, another 2 molar 
equivalents of triethylamine base catalyst were added. A solution of 2 molar equivalents acryloyl chloride per PEG chain in $\sim 20 \mathrm{~mL}$ dichloromethane was slowly dripped into the flask (about 1 drop every 3-5 seconds), and the reaction was stirred overnight. This second reaction step functionalized the remaining end of each PEG chain with an acrylamide group; an excess of acryloyl chloride was used to completely functionalize any additional ends that did not react with the cholesteryl chloroformate. Throughout the two-day synthesis, the reaction was kept at room temperature under inert atmosphere. By-product formation was minimized by careful attention to slow addition of the cholesteryl chloroformate and acryloyl chloride reactants to the reaction mixture. Following synthesis, dichloromethane was evaporated and the remaining solid was redissolved in tetrahydrofuran. The cholesterol-PEG-acrylamide (PEG-chol) product (Figure 1b) was isolated by filtration and evaporation of tetrahydrofuran followed by refrigeration and vacuum-drying.

${ }^{1} \mathrm{H}$ NMR analysis confirmed the presence of cholesterol and acrylamide end-groups on the PEG (see Figure S01 in the Supporting Information). Unfortunately, the presence of byproducts such as chol-PEG-chol or PEG-diacrylamide could not be distinguished using this technique. Chol-PEG-chol cannot covalently attach to the network and is removed via washing. Since only 1 molar equivalent of cholesterol per PEG chain is added, formation of large amounts of chol-PEG-chol would significantly reduce the PEG-chol yield and prevent much of the cholesterol from being incorporated into the networks. However, the data show structural and mechanical differences in the hydrogels with even small amounts of the synthesized product included, suggesting that the majority of cholesterol was attached and not washed out of the networks. Additionally, excess PEG-diacrylamide would act as a crosslinker in the formation of 100\% PEG-chol networks, greatly reducing their polymerization time and producing a network 
that is actually a combination of PEG and PEG-chol. Again, this seems negligible as the PEGchol networks took the longest to polymerize and showed properties significantly different from the PEG and PEG-chol copolymer networks. Thus, chol-PEG-chol and PEG-diacrylamide byproducts do not empirically show a large presence in the resulting networks.

Hydrogel preparation

Precursor stock solutions of PEG(4600)-diacrylate and PEG(1500)-chol were prepared by dissolving each macromonomer in chloroform at a concentration of $0.637 \mathrm{~g} / \mathrm{mL}$. The stock solutions were combined at different volume ratios to create the copolymer precursor solutions. Each precursor solution is described by the weight percent of mono-functional PEG-chol among the total solids in solution (i.e., 30\% PEG-chol $=0.3$ g PEG-chol for every 0.7 g PEGdiacrylate). Copolymer precursor solutions of 9\% PEG-chol to 90\% PEG-chol were prepared, equivalent to $1.8 \%$ to $18 \%$ cholesterol among the total solids by weight. 2-hydroxy-2methylpropiophenone photoinitiator was added to the precursor solutions at $1 \mathrm{wt} \%$ concentration with respect to the total solids content. Precursor solutions were then inserted between two glass slides separated by $0.5 \mathrm{~mm}$ thick Teflon spacers and exposed to a UV light source (365 $\mathrm{nm}$ at 10 $\mathrm{mW} / \mathrm{cm}^{2}$, UV chamber model ELC-500, Electro-lite Corporation, Danbury, CT). During UV exposure, crosslink junctions were created via photopolymerization of the telechelic PEGdiacrylate end groups. UV exposure time varied depending on the amount of PEG-chol in the precursor. Copolymer solutions with a majority of PEG-diacrylate ( $<50 \%$ PEG-chol) were more easily crosslinked, requiring only 5 minutes. Precursors with increasing amounts of PEG-chol were exposed sufficiently long to produce gels that could be easily removed from the glass slides and handled; this led to 8 minutes UV exposure for 60\% PEG-chol solutions and up to 13 minutes UV for $90 \%$ PEG-chol solutions. The resulting PEG-co-(PEG-chol) networks were 
placed in chloroform to wash overnight. The networks were subsequently washed in ethanol followed by deionized water, where they were then swollen to equilibrium.

To synthesize PEG(1500)-chol single polymer networks (100\% PEG-chol, no PEGdiacrylate), photoinitiator (1 wt\% with respect to PEG-chol) was added to the PEG-chol stock solution, and triethylene glycol dimethacrylate crosslinking agent was added at $10 \mathrm{~mol} \%$ with respect to PEG-chol. The precursor was placed between glass slides as described above and exposed to UV light for 20 minutes. The PEG-chol networks were washed in the same manner as the PEG-co-(PEG-chol) networks before equilibrium swelling in water.

Several variations of the PEG-co-(PEG-chol) networks were also prepared for comparison studies. Networks using PEG(3400)-chol were made as described above with the exception that the PEG-chol stock solution was prepared at $1.24 \mathrm{~g} / \mathrm{mL}$. This change was made so that the PEG(3400)-chol solution would be the same molar concentration as the PEG(1500)chol solution. Networks of PEG-diacrylate co-polymerized with mono-acrylated PEG(2000), referred to as PEG-co-(PEG(2000)), were synthesized identically to the PEG-co-(PEG-chol) networks, replacing PEG(1500)-chol ( $\left.M_{n} 2000 \mathrm{~g} / \mathrm{mol}\right)$ with mono-functional PEG(2000). All networks will be identified according to the wt $\%$ of the mono-functional PEG macromonomer with respect to the total polymer content (i.e., 30\% PEG-chol network derives from the $30 \%$ PEG-chol precursor solution). Small-angle $x$-ray scattering (SAXS) measurements

SAXS measurements were performed at the Stanford Synchrotron Radiation Lightsource (SSRL) on beamlines 1-4 and 4-2, similar to previous studies[27, 31]. Circular disks (15 mm diameter) of water-swollen hydrogel samples were cut out and placed into a sample holder with no x-ray windows. Two disks of each sample were stacked to decrease the x-ray beam 
transmission and increase the scattering intensity. During hydrogel drying measurements, a single disk sample was measured repeatedly over the course of a few hours. For measurements of networks swollen in organic solvent, the disks were assembled with excess solvent into a sample holder with Kapton windows, which was sealed shut using rubber o-rings.

Measurements were taken at different sample-to-detector distances, and the data were combined by shifting the intensities from one distance by an arbitrary factor to match the second. Separate measurements for background, Kapton and empty cell scattering were also taken with no beam and an empty sample holder with and without Kapton windows, respectively. Depending on the sample-to-detector distance, silver behenate, cholesteryl myristate and chicken tendon standards were used to calibrate the beam center. All image data was integrated using FIT2D software (European Synchrotron Radiation Facility, Grenoble, France).

Peak positions were determined by fitting the intensity as a function of the scattering vector, $q$, to a Lorentzian shape using a least squares fit. The position of the peak, $q_{\max }$, was related to the spacing between scatterers using Bragg's law,

$$
d=\frac{\lambda}{2 \sin \theta}=\frac{2 \pi}{q_{\max }}
$$

where $d$ is the average spacing between scattering centers, $\lambda$ is the x-ray wavelength, and $\theta$ is half of the scattering angle.

\section{Swelling measurements}

Hydrogel swelling measurements were completed by evaluating the weight of the hydrogels in their swollen $\left(W_{s}\right)$ and dry $\left(W_{d}\right)$ states[15, 33, 34]. After swelling to equilibrium in water, three small disks of each hydrogel were cut out, patted dry to remove surface water, and individually weighed. The disks were dried for three days in a vacuum desiccator to remove all water and then weighed again. The water content and equilibrium swelling ratio $\left(W_{s} / W_{d}\right)$ of each 
hydrogel were determined, and standard deviations for both were less than $\pm 2 \%$. The mass fraction $\left(W_{d} / W_{s}\right)$, or total polymer content, of each PEG-co-(PEG-chol) network was used to calculate the cholesterol mass fraction $\left(W_{c h o l} / W_{s}\right)$ of the hydrogels.

\section{Compressive mechanical testing}

Hydrogel compression samples were prepared using Teflon and neoprene spacers ranging from 2.32 to $3.09 \mathrm{~mm}$ thick. UV exposure time (5-15 minutes) was increased to secure complete polymerization and crosslinking of the thicker networks, with more time needed for higher PEGchol\% precursors. Extra washing steps were used to minimize macroscopic cracking during solvent transfer caused by osmotic swelling differences in the bulk and surface of the gel. After photopolymerization, the thick networks were washed in chloroform overnight followed by incremental washing steps: chloroform/ethanol (3:1 volume ratio), chloroform/ethanol (1:1), chloroform/ethanol (1:3), ethanol, ethanol/water (3:1), ethanol/water (1:1), ethanol/water (1:3) and water. The equilibrium-swollen hydrogels all had final thicknesses of $3.06 \pm 0.10 \mathrm{~mm}$. A 7 $\mathrm{mm}$ trephine punch was used to cut out cylindrical samples from the hydrogel slabs $3.1 \mathrm{~mm}$ in height and $7 \mathrm{~mm}$ in diameter; a similar height-to-diameter ratio was used previously for compression testing on comparable hydrogels[22, 27, 31].

Compressive mechanical testing was performed using an Instron Universal Materials Testing Machine (Instron model \#5844, Norwood, MA, USA) with Bluehill 2 software. A strain rate of $1 \mathrm{~mm} / \mathrm{min}$ was applied to each hydrogel cylinder until breakage, indicated by a sudden decline in the measured stress. The measured load and compressive extension were collected and used to calculate engineering stress, $\sigma_{\text {eng }}$ (normal force over initial cross-sectional area), as a function of deformation, $\alpha$ (ratio of compressed to initial gel thickness), for 8 to 16 samples of each type of hydrogel. 


\section{Results and discussion}

SAXS from networks with low PEG-chol content

SAXS of the PEG(4600)-co-(PEG(1500)-chol) networks (<50\% PEG-chol) swollen in both organic and aqueous solvent was employed to interpret structural differences on the nanoscale as cholesterol was introduced into the polymer networks. After synthesis in chloroform, PEG-co-(PEG-chol) networks were transferred to water for SAXS measurements, and then the same networks were washed in ethanol and swollen to equilibrium in tetrahydrofuran (THF) for another set of SAXS measurements. Polymer networks were swollen in THF because it is less toxic and volatile than chloroform for open air SAXS experiments but has similar solubility of PEG chains[35]. X-ray scattering contrast between hydrophobic elements in the network and the organic solvent was verified using the end-linked PEG networks (0\% PEG-chol) that display a scattering peak corresponding to their weakly ordered, hydrophobic crosslink junctions[27] (see Figure S02 in the Supporting Information).

Scattering curves for the PEG-co-(PEG-chol) networks swollen to equilibrium in organic and aqueous solvent are shown in Figure 2. In THF, the networks exhibit peak-like scattering at very low PEG-chol concentrations (0\% and $9 \%)$ that develops into broad shoulders for increasing amounts of incorporated PEG-chol. The occasional presence of a small peak around $\mathrm{q}$ $\approx 3.6 \mathrm{~nm}^{-1}$ results from excess background scattering of the Kapton windows and is not indicative of any structural features. In contrast, scattering peaks were measured for all the PEGco-(PEG-chol) networks swollen to equilibrium in water regardless of PEG-chol concentration. SAXS curves analogous to those in Figure 2 were observed from PEG-co-(PEG-chol) networks swollen in acetonitrile as well as networks synthesized in THF and measured in THF and water (see Figures S03 and S04 in the Supporting Information), thereby demonstrating that differences 
between network features in organic and aqueous solvent must result from self-assembly or molecular rearrangement within the polymer network during solvent processing. To maintain a random distribution of the hydrophobic cholesterol throughout the network would be entropically unfavorable for the aqueous system, and with no significant steric hindrances to prevent rearrangement, it is reasonable for the cholesterol to re-organize into a more energetically favorable aggregated conformation during solvent transfer to water.

(a)
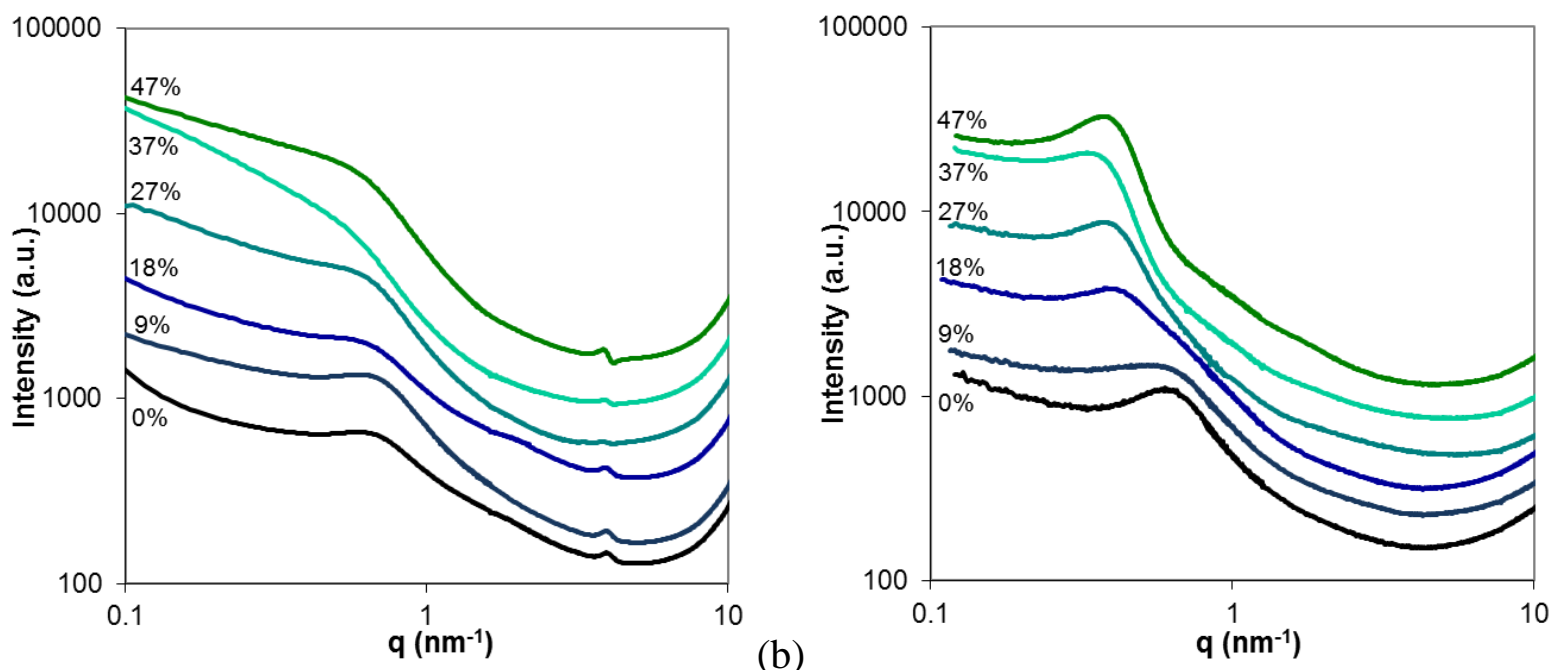

Figure 2. SAXS curves for PEG-co-(PEG-chol) networks swollen in (a) THF and (b) water. Scattering intensities are in arbitrary units (a.u.) and have been offset for clarity. The percentages shown represent the amount of incorporated PEG-chol (wt PEG-chol/wt total solids), with 0\% PEG-chol representing a standard PEG network with no cholesterol.

Scattering in THF is representative of the network structure during synthesis in organic solvent. For 0\% PEG-chol, or a standard PEG network, the broad peak signifies weak ordering of the scattering groups, in this case the hydrophobic crosslink junctions, and the peak position correlates with the crosslink junction spacing as seen previously[27]. With the addition of cholesterol, the broad shoulder patterns indicate a random distribution of scattering groups throughout the PEG-co-(PEG-chol) networks. The lack of any major shift in the position of the shoulders implies that the size of the scattering objects remains constant for all PEG-chol 
concentrations. Incorporating cholesterol into the PEG network introduces a second source of hydrophobic scattering; the PEG linker separates cholesterol from the crosslink junction, but should not be long enough to allow cholesterol to interfere with nearby crosslinks. The cholesterol is somewhat free to move around in the network, and in organic solvent where it is highly soluble, it is likely to situate itself randomly throughout the network between crosslink junctions. The PEG-co-(PEG-chol) crosslink junctions should still remain weakly ordered, however, because the networks are composed of over 50\% end-linked PEG.

Conversely, the correlation peaks in the water-swollen PEG-co-(PEG-chol) gels suggest a weakly ordered formation of the hydrophobic crosslink junctions and cholesterol domains. The peak position also shifts to lower q, or larger spacing, as the amount of PEG-chol in the network is increased. The randomly distributed cholesterol molecules are believed to collapse into the crosslinks upon transition to aqueous solvent, in which case the scattering peak would still correlate to crosslink junction spacing. Such an arrangement would be entropically favored by creating larger hydrophobic groups throughout the swollen network, thus releasing the tightly ordered water structure surrounding each cholesterol molecule. Alternatively, the cholesterol molecules attached to nearby crosslinks through their PEG-linkers could self-assemble into clusters between the crosslink junctions. If this were the case, the distance between scattering groups would decrease and the correlation peak would shift to higher q with increased cholesterol content, contrary to the results. The increase in crosslink junction spacing as the amount of cholesterol rises indicates a slight extension of the PEG chains, likely to accommodate for the additional bulkiness from cholesterol aggregation at the crosslinks.

The schematic in Figure 3 summarizes our structural interpretation as the PEG-co-(PEGchol) networks are initially formed and then undergo solvent exchange. During 
photopolymerization, the functional end-groups in the well-mixed organic precursor solution react to form dense, hydrophobic crosslink junctions. The end-linked PEG chains form a weakly ordered array of crosslinks, while the mobile cholesterol molecules are randomly distributed among the crosslinks throughout the polymer network. Solvent exchange to form a hydrogel swollen in water causes the cholesterol to collapse into the crosslink junctions.
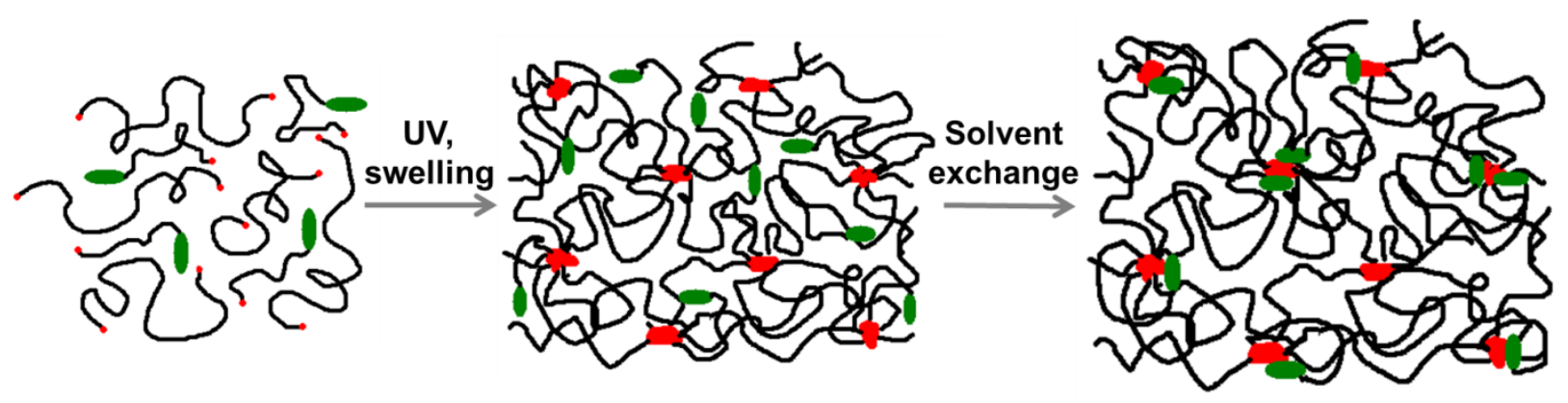

Figure 3. Schematic depicting the network structure of PEG-co-(PEG-chol) networks with low PEG-chol content $(<50 \%)$ as they are synthesized in organic solvent and transferred to water. Photopolymerization of PEG and PEG-chol macromonomers (left) creates a network with a random distribution of hydrophobic groups (center; ordered crosslinks, red, and randomlyarranged cholesterol molecules, green). Cholesterol collapses into the crosslink junctions after aqueous solvent exchange (right).

\section{SAXS from networks with high PEG-chol content}

Hydrogels with higher PEG-chol concentrations were analyzed to investigate the selfassembly behavior of cholesterol as the network transitions from being a tightly crosslinked PEG network with inserted PEG-chol to a network composed mostly of PEG-chol monomers loosely crosslinked by PEG-diacrylate. Figure 4 displays the SAXS behavior for water-swollen PEG-co(PEG-chol) hydrogels containing the full range of PEG to PEG-chol ratios, from PEG (0\% PEGchol) to a $100 \%$ PEG-chol network made with a small crosslinker. Below 60\% PEG-chol, the SAXS curves have a single peak correlating to the PEG crosslink spacing; larger amounts of PEG-chol (30-60\%) shift the peak to lower q as a result of cholesterol collapse into the crosslink junctions extending the PEG chains. A structural transition occurs above $60 \%$ PEG-chol as the 
original peak shifts back to higher $\mathrm{q}$ and a second peak develops. The appearance of two distinct peaks is most obvious for the $100 \%$ PEG-chol network.

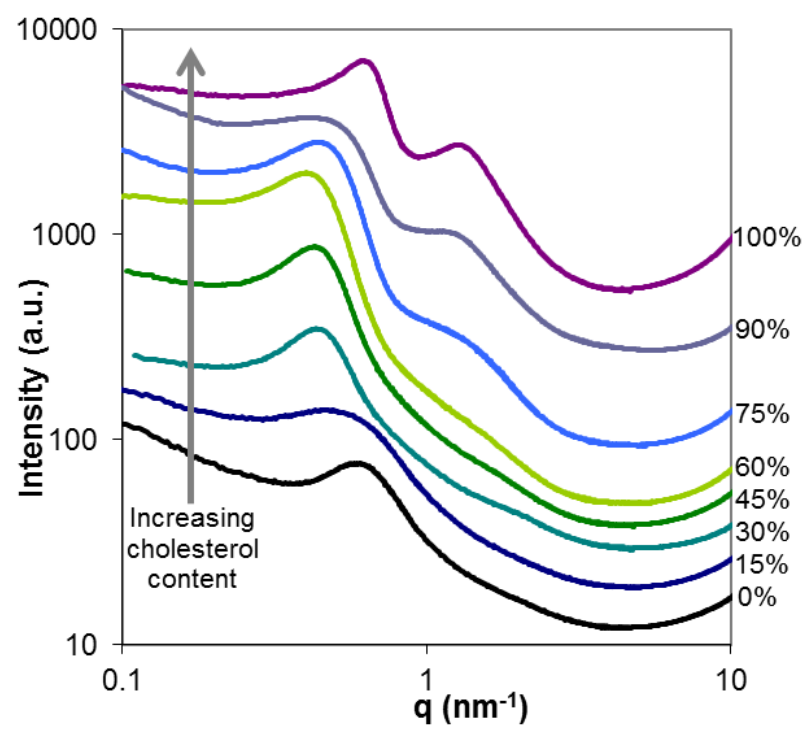

Figure 4. SAXS curves from PEG-co-(PEG-chol) networks swollen in water as the ratio of PEG-chol to PEG is increased from 0\% (PEG single network) to 100\% (PEG-chol single network).

Peak positions for the curves in Figure 4 were determined, and the corresponding $d$ spacings are shown in Table 1. The distance between crosslinks in the PEG network was 10.7 $\mathrm{nm}$, a spacing similar to that measured previously[27]. Comparison with the theoretical end-toend distance, $R$, of a PEG(4600) chain shows that the spacing is between that of a self-avoiding random walk in three dimensions $(7.6 \mathrm{~nm})$ and two dimensions $(14.3 \mathrm{~nm})$ [27]. End-to-end distance was calculated using equation 2 where $b$ is the Kuhn length for PEG $(0.76 \mathrm{~nm}), N$ is the number of Kuhn segments, and $v$ is the scaling exponent for a self-avoiding random walk in a good solvent ( $v=0.588$ for three dimensions and $v=0.75$ for two dimensions) [27, 36, 37].

$$
R \approx b N^{v}
$$

A continuous increase in the crosslink spacing was detected with the gradual addition of PEGchol into the network until reaching a spacing of $15.8 \mathrm{~nm}$ at $60 \%$ PEG-chol. A 3-5 nm increase 
in the crosslink spacing from cholesterol collapsing into the junctions seems reasonable considering the configurational changes that must occur during its rearrangement.

Table 1. SAXS $d$-spacing for water-swollen PEG networks co-polymerized with monofunctional PEG-chol or PEG(2000).

\begin{tabular}{ccc}
\hline \multirow{2}{*}{$\begin{array}{c}\text { Mono-functional PEG\% } \\
\text { (wt/total solids wt) }\end{array}$} & \multicolumn{2}{c}{$d$-spacing (nm) } \\
\cline { 2 - 3 } & PEG-co-(PEG-chol) & PEG-co-(PEG(2000)) \\
\hline 0 & 10.7 & 10.7 \\
15 & 13.6 & 11.2 \\
30 & 14.5 & 11.6 \\
45 & 14.8 & 12.2 \\
60 & 15.8 & 14.4 \\
75 & 14.4 & No peak \\
90 & 15.4 & No gel \\
100 & 10.2 & No gel \\
\hline
\end{tabular}

Above 60\% PEG-chol, the $d$-spacing of the main peak decreases as the second peak emerges at higher q. One exception is the distance measured for the 90\% PEG-chol hydrogel, but the broadness of the peaks exhibited by that network makes determining peak positions difficult. A shift of the principal peak back to higher $q$ indicates that scattering is no longer dominated by the weakly-ordered crosslinks but rather from a different cholesterol aggregation structure with smaller spacing that becomes even more dominant at concentrations above $75 \%$ PEG-chol. The differences in $d$-spacing between the first and second peaks of the 100\% PEGchol network, $10.2 \mathrm{~nm}$ and $5.0 \mathrm{~nm}$ respectively, signify lamellar ordering of the scattering groups within the hydrogel[38, 39]. At low PEG-chol concentrations, the network contains dense, hydrophobic crosslinks and the cholesterol is sparsely distributed, so it is much more likely for cholesterol to aggregate with the crosslink junctions rather than with other cholesterol molecules. In contrast, networks with high PEG-chol content will no longer contain dense crosslinks due to 
low amounts of PEG-diacrylate. Instead, reaction of the PEG-chol acrylamide end-groups will form a hydrophobic polymer backbone with sparsely distributed PEG-diacrylate. The cholesterol groups should be located much closer together such that they can more easily locate one another and assemble together during transfer to aqueous solvent. Cholesterol can also exhibit liquid crystalline behavior[40], so stacking into lamellar-like regions is quite reasonable.

Besides adding cholesterol to the hydrogel, replacing PEG-diacrylate with monofunctional PEG-chol removes effective network chains and introduces dangling end-groups into the network. In order to confirm that structural features observed in the PEG-co-(PEG-chol) hydrogels are due to cholesterol self-assembly and not just the effects of increased dangling PEG chains, mono-acrylated PEG(2000) was substituted for PEG-chol to form PEG-co-(PEG(2000)) networks. SAXS results for the PEG-co-(PEG(2000)) hydrogels are shown in Figure 5, and the $d$-spacings are presented in Table 1 . The crosslink spacing increased slightly from $10.7 \mathrm{~nm}$ for the standard PEG network up to $12.2 \mathrm{~nm}$ for $45 \%$ PEG(2000), showing that the addition of dangling PEG chains within the hydrogel has a measureable, but minor, effect. As was observed in the PEG-co-(PEG-chol) networks, 60\% PEG(2000) appears to be a transition point for the hydrogel structure. At this ratio, the scattering peak broadens and the crosslink spacing increases to $14.4 \mathrm{~nm}$ consistent with a plethora of swollen, dangling PEG end-groups that substantially loosen the weakly ordered structure. Above this concentration, the ordered crosslinks are completely disrupted, as a scattering shoulder indicating a random distribution of scattering groups was observed for $75 \%$ PEG(2000) networks. 


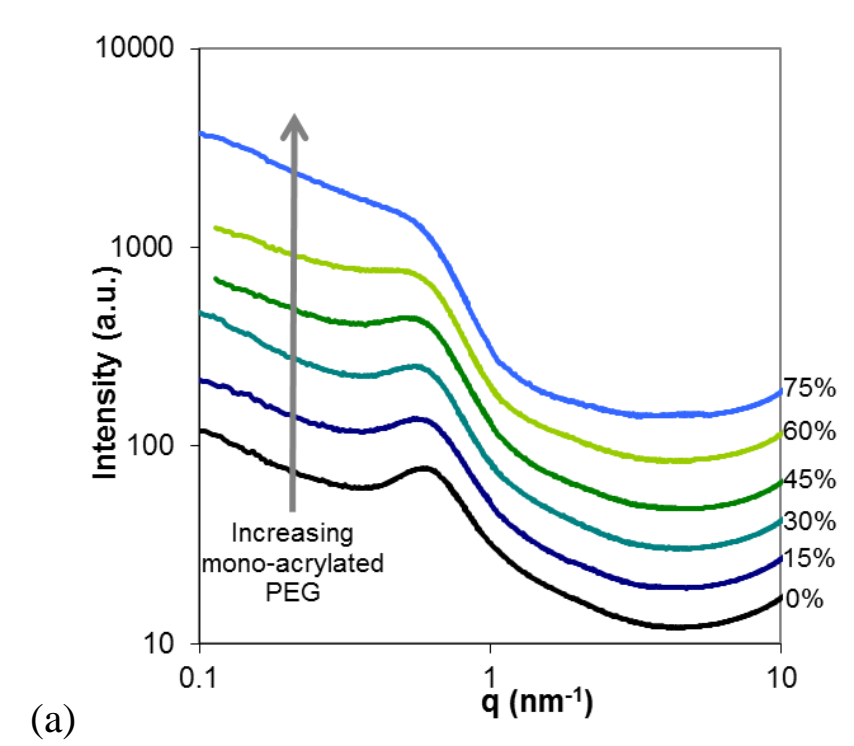

(a)

(b)
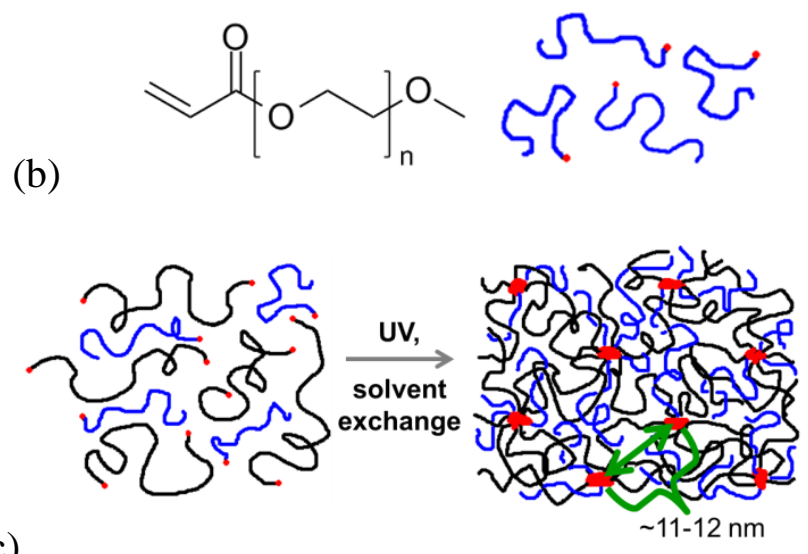

(c)

Figure 5. (a) SAXS curves from PEG-co-(PEG(2000)) networks swollen in water with varying concentration of mono-functional PEG(2000) (0\% to $75 \% \mathrm{wt} /$ total solids wt). (b) Chemical structure of mono-acrylated PEG ( $\mathrm{n} \approx 45$ for $\left.M_{n} 2000 \mathrm{~g} / \mathrm{mol}\right)$ and cartoon graphic where blue lines represent PEG chains and red circles represent hydrophobic acrylate end-groups. (c) Schematic depicting the network structure of PEG-co-(PEG(2000)) networks as they are synthesized in organic solvent and transferred to water. A slight increase in crosslink spacing occurs with increased PEG(2000) content due to dangling end-groups within the hydrogel.

Side-by-side comparison of the measured $d$-spacing for PEG-co-(PEG-chol) and PEG-co(PEG(2000)) networks in Table 1 shows that the PEG-co-(PEG-chol) networks all have greater spacing between crosslink junctions. While the addition of dangling PEG chains has only a small effect on network structure, it is clear that cholesterol rearrangement has a substantial influence on the hydrogel morphology. Interestingly, both network compositions show a 
structural transition between $60 \%$ and $75 \%$ incorporated mono-functional PEG. This may be the point where the network no longer contains the critical amount of PEG-diacrylate chains needed to maintain a weakly ordered crosslink structure; instead, the PEG-diacrylate simply acts as a loose crosslinker for the photopolymerized chains of mono-functional PEG-chol or PEG(2000). At the higher ratios of PEG-chol, the PEG-co-(PEG-chol) networks appear to contain a local lamellar-like structure that develops as a result of this morphological transition. Additional studies on the $100 \%$ PEG-chol networks, which exhibited the clearest scattering, were performed to further investigate this possible conformation.

\section{SAXS from PEG-chol networks}

The scattering measurements in Figure 2 indicating random cholesterol distribution during organic synthesis were only performed at lower PEG-chol ratios. Thus, $100 \%$ PEG-chol networks were analyzed in THF to explore any structural features that affect interpretation of the water-swollen hydrogel structure. SAXS measurements were taken directly after synthesis, after solvent transfer to water and following a second solvent exchange cycle in THF and water to observe if structural features were maintained (Figure 6). In THF, the networks exhibit one broad shoulder indicating a random distribution of cholesterol throughout the gel, similar to the PEG-co-(PEG-chol) networks with low PEG-chol content. As expected, the water-swollen PEGchol gels display two distinct peaks with peak positions identical to those in Figure 4 . The scattering curves are equivalent for each solvent condition regardless of the solvent processing steps that came beforehand. The results demonstrate that cholesterol is distributed randomly during network formation in organic solvent but then self-assembles into an ordered configuration when the network is swollen in water; the molecular rearrangement is both reversible and repeatable through solvent exchange. The development of the self-assembled 
structure after polymerization and crosslinking of the network suggests that the ordering is restricted to small, local domains throughout the hydrogel.

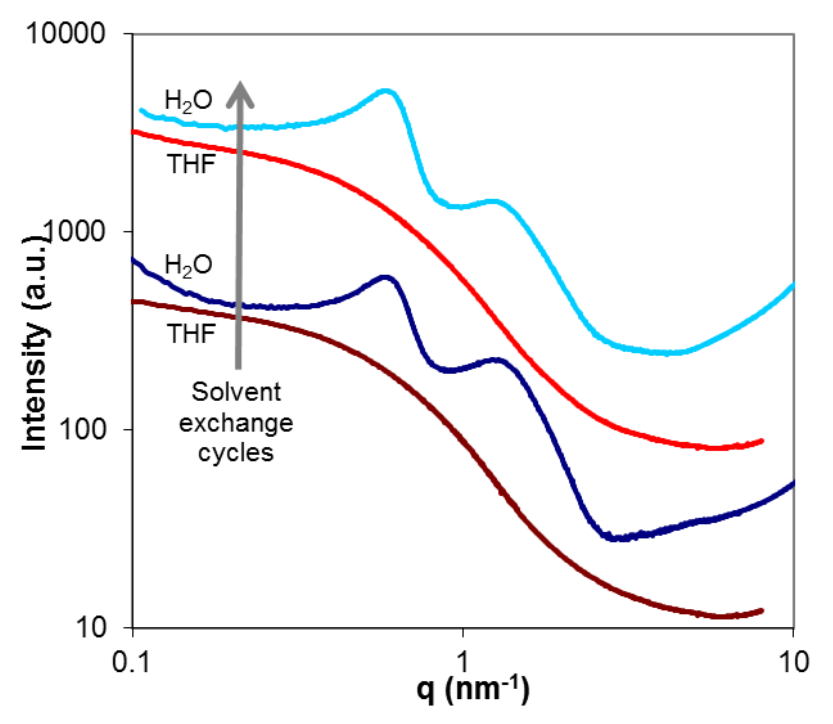

Figure 6. SAXS curves of a PEG-chol network made in chloroform and measured during multiple solvent exchange cycles between THF and water.

Since the two peaks observed in the hydrogel network are not carried over from any preexisting features, it is probable that they are first and second order peaks of a single ordered arrangement rather than the result of two separate ordered configurations on different length scales. The 2:1 ratio of $d$-spacing signifies lamellar-like organization of cholesterol domains within the PEG-chol network[38, 39]. Cholesterol assembly into lamellar-like sheets is not surprising given the nature of cholesterol and the composition of the network. For example, as a liquid crystalline material, cholesterol easily stacks into sheets[40]. Moreover, the polymer backbones are expected to be centered between the cholesterol sheets, with PEG side chains emanating from either side connected to the cholesterol clusters. The position of the SAXS peak corresponds to the distance between the centers of the cholesterol layers, which should be similar to twice the end-to-end distance of the PEG chain plus the length of the long axis of a cholesterol molecule. Using equation 2 for a three-dimensional self-avoiding random walk combined with 
the measured length of cholesterol[41], the calculated theoretical lamellar spacing is $9.4 \mathrm{~nm}$, only slightly smaller than the measured spacing of $10.2 \mathrm{~nm}$ for the PEG-chol hydrogels.

To verify this conformation, the length of the PEG-linker in the PEG-chol monomer was altered. If the cholesterol assembles into stacked aggregates ordered like lamellar sheets, then the spacing of the cholesterol groups will relate to the length of the PEG chains attaching it to the polymer backbone. Increasing the length of the PEG chains, for instance, should increase cholesterol spacing and cause both peaks to shift to lower q in the SAXS pattern. PEG(3400)chol monomers were synthesized for development of a 100\% PEG(3400)-chol network; however, the precursor solution did not form a fully crosslinked gel that could be handled, even with extended UV exposure. As an alternative, a PEG-co-(PEG-chol) network with 88\% PEG(3400)-chol was synthesized for comparison to the $90 \%$ and $100 \%$ PEG(1500)-chol networks (Figure 7). The 88\% PEG(3400)-chol network displays a peak at significantly lower $\mathrm{q}$ than the hydrogels consisting of PEG(1500)-chol, with a corresponding $d$-spacing of $20.6 \mathrm{~nm}$. The network also exhibits a shoulder at the position of the first PEG(1500)-chol peak, around a $d$-spacing of 10-11 nm, and a second faint shoulder near the position of the second PEG(1500)chol peak, which has a $d$-spacing of $5 \mathrm{~nm}$. Thus, the $88 \%$ PEG(3400)-chol network, with PEGlinkers twice the length of the $100 \%$ PEG(1500)-chol network, demonstrates a first, second and faint third moment in the SAXS pattern with corresponding scattering distances twice the size of those measured for the PEG(1500)-chol network. The $d$-spacing ratio between the first and second (and third) moment features is (3):2:1 for both networks. Together, the peak shift to larger spacing and the constant ratio of the peaks confirm the presence of lamellar-like spacing within the self-assembled cholesterol domains. 


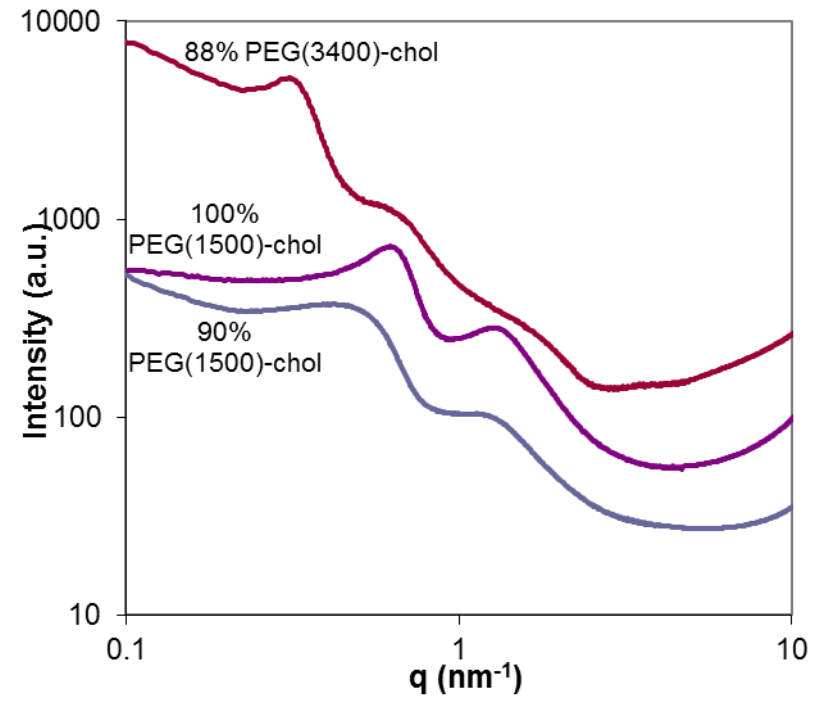

Figure 7. X-ray scattering of a PEG-co-(PEG-chol) network made using PEG(3400)-chol monomers at high concentration compared to the high-cholesterol content networks made from PEG(1500)-chol monomers.

A schematic of the proposed PEG-chol network morphology is presented in Figure 8.

Following homogeneous network synthesis, the limitations in mobility of the PEG side chains due to their attachment to the polymer backbone restrict the assembly of cholesterol such that small, local domains with lamellar-like ordering are formed. Amorphous regions of PEG and disordered cholesterol aggregates surround the phase-separated domains.

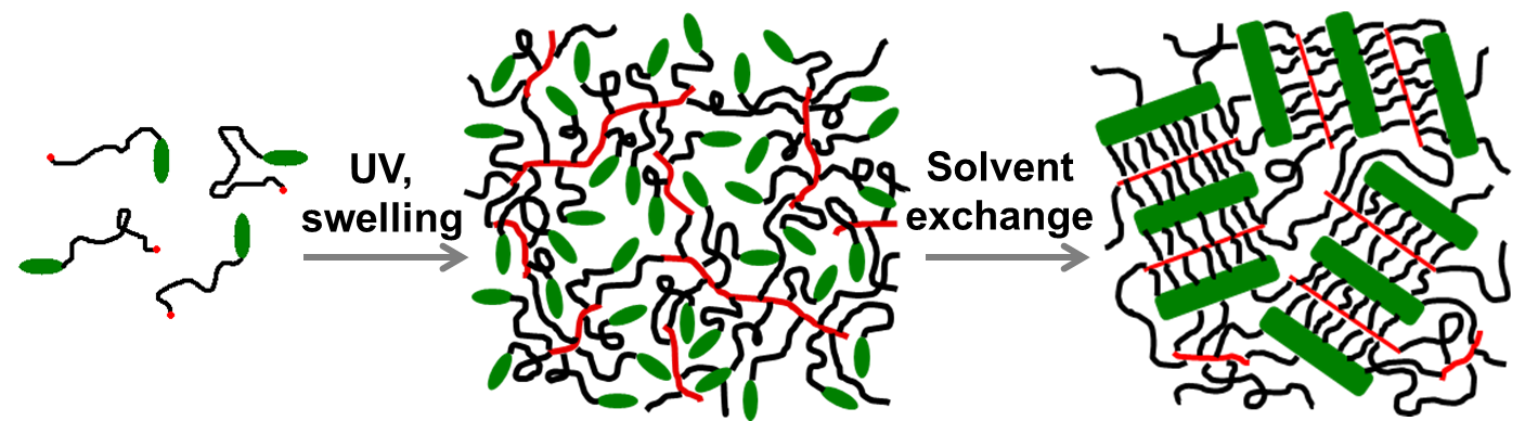

Figure 8. Schematic depicting the morphology of 100\% PEG-chol networks as they are synthesized in organic solvent and transferred to water. PEG-chol macromonomers (left) form a loosely-crosslinked network with randomly-distributed cholesterol side chains (center) upon photopolymerization. Cholesterol self-assembles into lamellar-like domains during aqueous solvent exchange (right). 


\section{Structural dependence on cholesterol content}

Confirmation of the organized nanostructure at high PEG-chol to PEG ratios completes our understanding of cholesterol self-assembly in the water-swollen PEG-co-(PEG-chol) networks. The schematic in Figure 9 demonstrates the three structural configurations observed as the ratio is increased from $0 \%$ to $100 \%$ PEG-chol. Without any cholesterol incorporation, the structure of the photopolymerized, end-linked PEG network is characterized by weak ordering of the dense, hydrophobic crosslink junctions. Addition of PEG-chol does not significantly alter the crosslink ordering below 60\% PEG-chol; however, as cholesterol collapses into the crosslinks, even denser hydrophobic moieties are generated and the PEG chains become stretched. Above 75\% PEG-chol, cholesterol starts to aggregate with itself, and the hydrogel structure slowly transitions into a phase-separated morphology of lamellar-like ordered domains surrounded by amorphous, disordered PEG chains and cholesterol clusters. The lamellar ordering becomes much more distinct with the elimination of long PEG-diacrylate chains, replaced by a small triethylene glycol crosslinker, as demonstrated by the well-defined peaks in the SAXS pattern of the 100\% PEG-chol network. Additional investigation on the corresponding macroscopic properties of the gels was performed for verification of the proposed micro-morphology. 


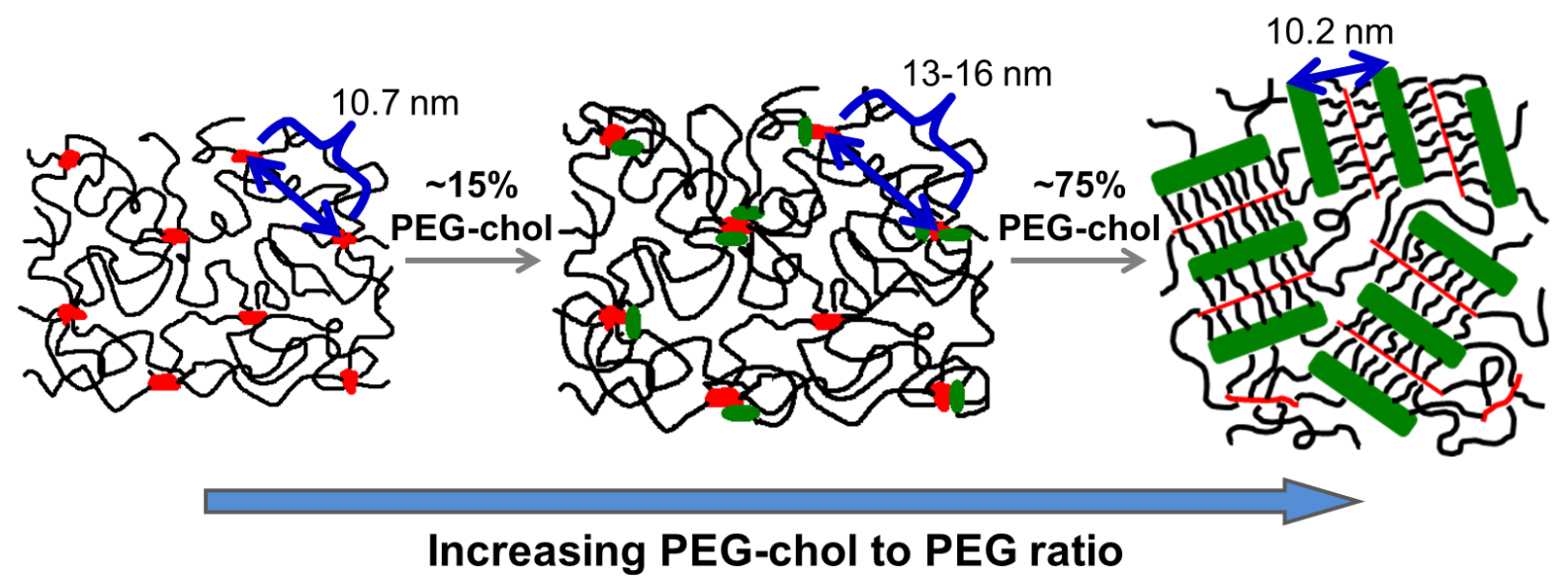

Figure 9. Schematic of the hydrogel morphology for water-swollen PEG-co-(PEG-chol) networks of varying composition, from $0 \%$ to $100 \%$ PEG-chol. PEG chains are drawn in black, the polymerized PEG end-groups are shown in red, and cholesterol groups are shown in green.

\section{Swelling measurements}

Incorporating cholesterol into hydrogels via the PEG-chol linker introduces competitive swelling effects. Although the presence of cholesterol would be expected to stimulate less swelling due to its hydrophobic nature, replacement of PEG-diacrylate chains with monofunctional PEG-chol loosens the crosslinking and entanglement structure and adds dangling PEG chains, both of which promote water uptake. In addition, the self-assembled cholesterol morphology may affect PEG swelling capabilities within the networks. To separate these competing influences, swelling measurements were performed on both PEG-co-(PEG-chol) and PEG-co-(PEG(2000)) hydrogels. Figure 10 shows the measured equilibrium swelling ratio, Q, which represents the weight ratio of water to polymer in the gels. As expected, the swelling ratio of the PEG-co-(PEG(2000)) networks gradually increases with the incorporation of monofunctional PEG. This corresponds to the small but gradual increase in crosslink spacing that was measured via SAXS. With increasing amounts of PEG(2000) replacing PEG-diacrylate, the number of PEG chains acting as effective crosslinkers decreases and the constricted network loosens. Although the PEG-co-(PEG(2000)) hydrogels do not undergo any major structural 
transitions like those observed in the PEG-co-(PEG-chol) networks, the disappearance of weakly ordered crosslinks was observed in the scattering profile for the 75\% PEG(2000) composition. Consequently, the transition to this even less restricted structure resulted in the abrupt increase in the equilibrium swelling ratio.

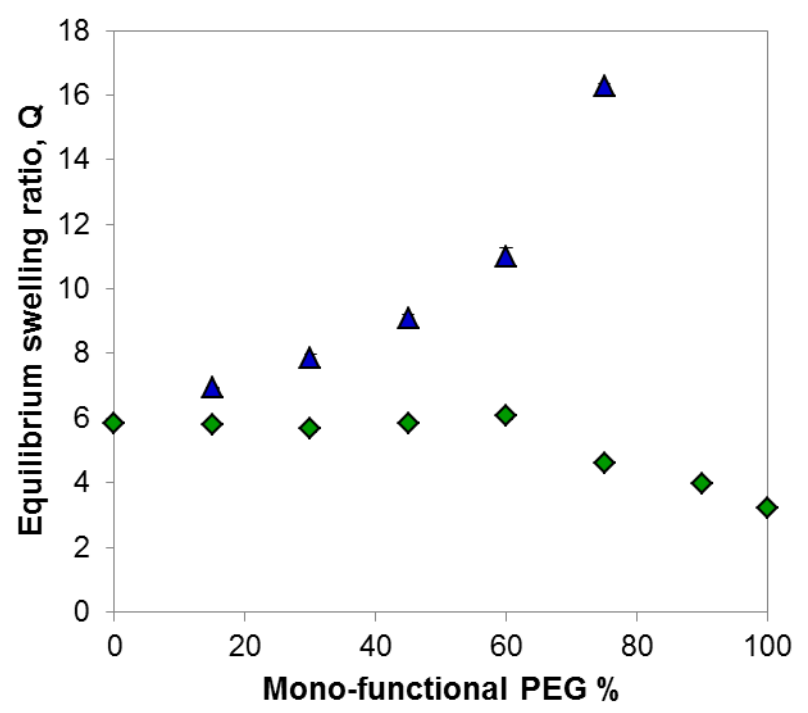

Figure 10. Equilibrium swelling ratio, Q, for PEG-co-(PEG-chol) (green diamond) and PEG-co(PEG(2000)) (blue triangle) networks of varying composition. The percent of mono-functional PEG refers to the weight fraction of PEG-chol or PEG(2000) among the total polymer solids in the network.

The swelling ratios of the PEG-co-(PEG(2000)) networks show the swelling trend expected for a gradual inclusion of mono-functional, dangling PEG chains into the end-linked PEG network. However, comparison with the measured swelling ratios of the PEG-co-(PEGchol) hydrogels reveals that this is not the dominant factor in those networks. Instead, the PEGco-(PEG-chol) networks exhibit identical swelling ratios with increasing PEG-chol content up to the structural transition between $60 \%$ and $75 \%$ PEG-chol, above which the water-to-polymer ratio gradually decreases. The unfluctuating swelling behavior below $60 \%$ PEG-chol must result from a balance of hydrophilic and hydrophobic swelling forces in the PEG-co-(PEG-chol) hydrogels that is independent of the PEG-chol composition. Every dangling PEG chain that 
increases swelling is attached to a cholesterol group that reduces it; greater crosslink spacing from more stretched and swollen PEG chains is balanced, and caused by, cholesterol aggregation to form larger hydrophobic crosslink junctions.

Above 60\% PEG-chol, the swelling ratio decreases with increasing amounts of PEGchol, correlating with the structural transition to meso-ordered cholesterol domains in the network. Self-assembly of cholesterol into stacked sheets creates large hydrophobic domains that are spaced closer together than the dense crosslinks at lower PEG-chol concentrations, meaning that less water absorbs between the hydrophobic network aggregates. Limited water absorption within the lamellar-like cholesterol structures leads to a decrease in the equilibrium swelling ratio as the presence of these domains in the network grows.

The mass fraction of cholesterol within each swollen PEG-co-(PEG-chol) network was calculated, as shown in Figure 11. Given the substantial alterations to the hydrogel structure that stem from cholesterol self-assembly, the actual mass fraction of cholesterol in the water-swollen networks is surprisingly small. The PEG-co-(PEG-chol) hydrogels are all composed of less than 7\% cholesterol by swollen mass, and the structural transition to lamellar-like domains and subsequent network de-swelling occurs at a mass fraction of 0.02 , or only $2 \%$ cholesterol in the swollen gel. For a single chain polymer amphiphile in solution, 2-7\% hydrophobic content would be unlikely to produce a self-assembled lamellar structure[42]. Instead, the amphiphiles would be more likely to adopt micellar or hexagonal ordering, which effectively hides the hydrophobic groups from having to interact with the aqueous solvent. However, the selfassembly of PEG-chol amphiphiles is significantly restricted by their attachment to the crosslinked network, which inhibits rearrangement to a more favorable morphology. The stability of lamellar domains in a similar PEG system was previously observed when block co- 
polymers mixed with an aqueous PEG-diacrylate precursor solution self-assembled into lamellar meso-structured domains, which were subsequently integrated into the hydrogel network[43]. Lamellar ordering occurred at block co-polymer concentrations as low as $10 \mathrm{wt} \%$, substantially lower than the $75 \mathrm{wt} \%$ concentration needed to form lamellar sheets in pure water[39, 43]. The results showed that the PEG chains stabilized lamellar meso-ordering, and this same effect may occur in the PEG-co-(PEG-chol) hydrogel networks.

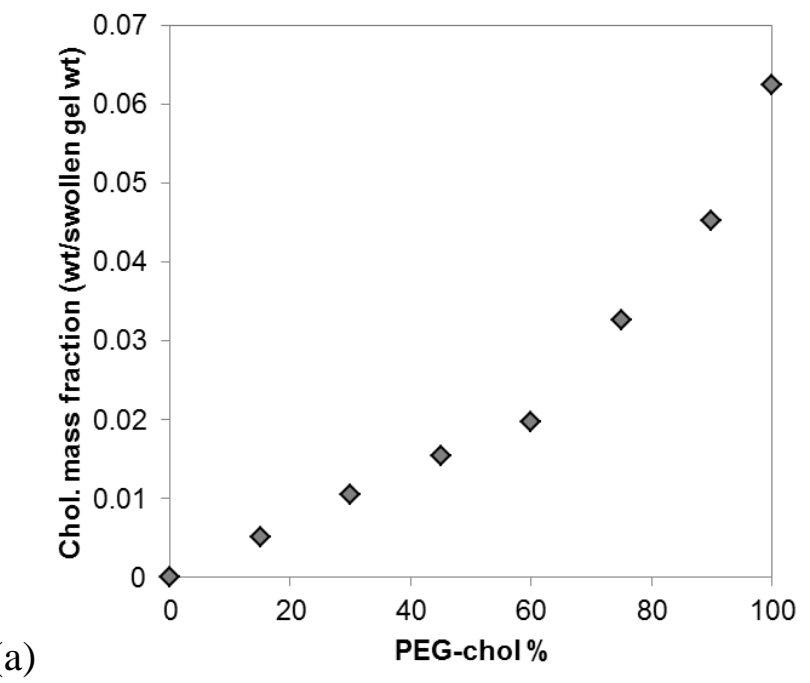

(a)

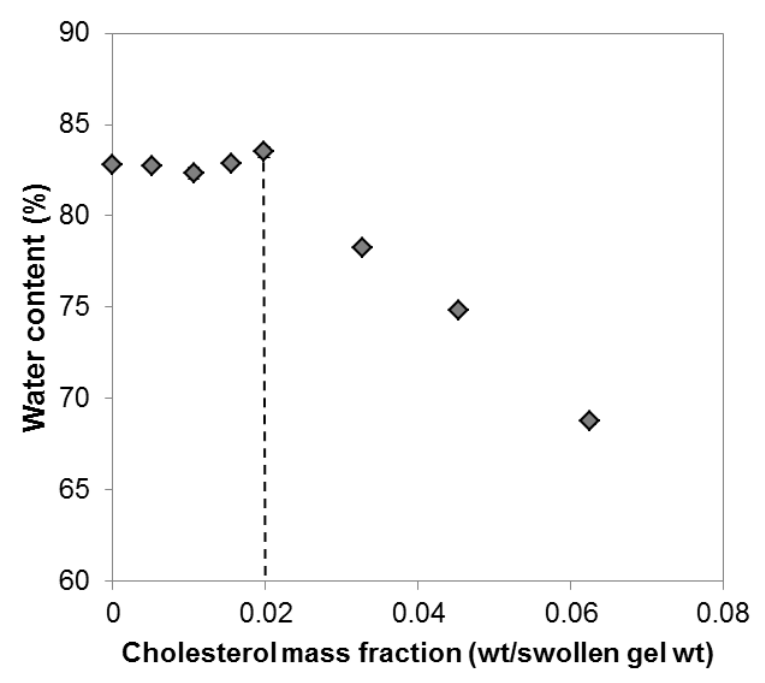

Figure 11. (a) The mass fraction of cholesterol in the water-swollen network for PEG-co-(PEGchol) hydrogels with $0 \%$ to $100 \%$ PEG-chol. (b) Water content as a function of the cholesterol mass fraction in the swollen gel. The dashed line indicates that the structural transition to lamellar-like domains occurs at a mass fraction of 0.02 , or $2 \%$ cholesterol. 


\section{Compressive mechanical testing}

Mechanical properties of the PEG-co-(PEG-chol) networks were investigated using compressive mechanical testing. Generally, hydrophobic groups would be expected to provide strength or toughness to the water-swollen network[18]; however, the cholesterol groups are attached to elastically ineffective dangling PEG chains, thus making it unclear what influence their incorporation might have on mechanical properties. Figure 12 shows the compressive stress data for uniaxial compression of the PEG-co-(PEG-chol) hydrogel networks plotted as a function of inverse deformation, $1 / \alpha$. Among the hydrogels with lower PEG-chol content, small differences in the compressive response are observable. The most notable change occurs between $0 \%$ and $15 \%$ PEG-chol networks, where the collapsed cholesterol increases the stress incurred by the network during deformation. Bigger changes in the stress behavior occur at high PEG-chol fractions as the network morphology transitions from ordered crosslinks to selfassembled lamellar-like domains. The structural alteration significantly impacts the ductility of the hydrogels, with the $90 \%$ and $100 \%$ PEG-chol networks undergoing 40-50\% more deformation and 6-7 times more stress than the 60\% PEG-chol hydrogels before breakage. 
(a)

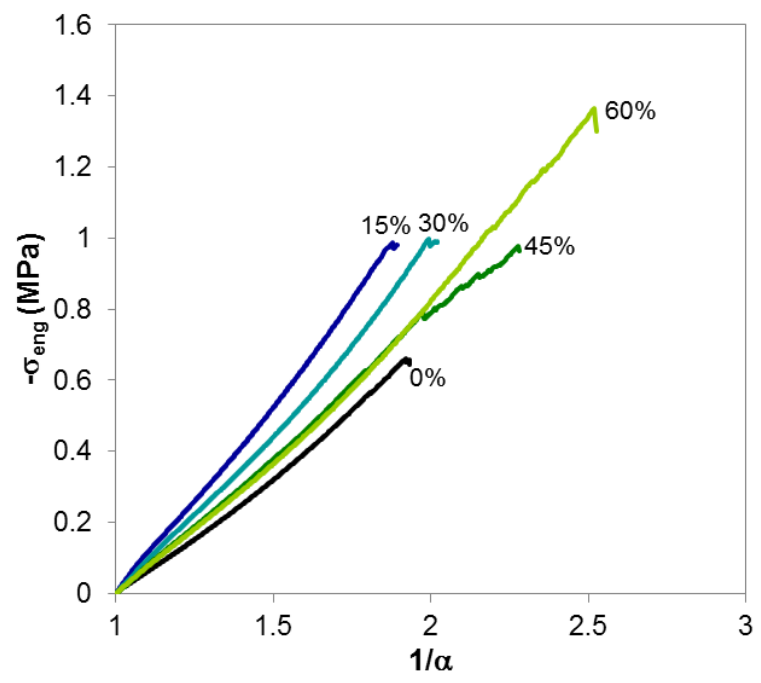

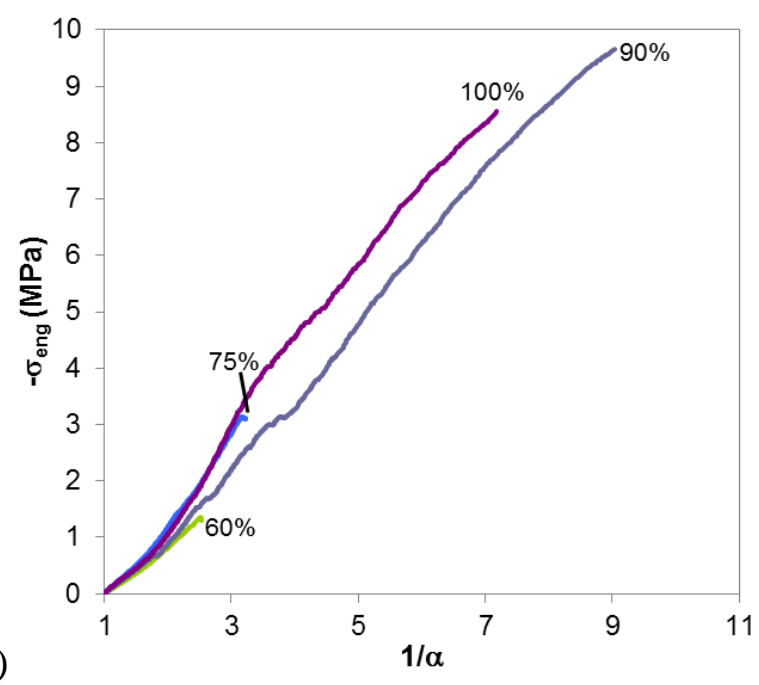

Figure 12. Compressive engineering stress, $\sigma_{\text {eng }}$, plotted against the inverse deformation, $1 / \alpha$, for water-swollen PEG-co-(PEG-chol) networks with varying fractions of incorporated PEGchol. (a) Data for 0-60\% PEG-chol networks with cholesterol aggregated at the crosslink junctions. (b) Data for 60-100\% PEG-chol networks with lamellar-like domains of selfassembled cholesterol. The most representative curve from each PEG-chol concentration (8-16 samples) was chosen and plotted.

Mechanical behavior of the PEG-co-(PEG-chol) networks is further elucidated by plotting the reduced nominal stress, $\sigma^{*}$, against $1 / \alpha$, as described by the Mooney-Rivlin equation[36, 44-46]:

$$
\sigma^{*}=\frac{\sigma_{e n g}}{\alpha-\left(1 / \alpha^{2}\right)}=2 C_{1}+\frac{2 C_{2}}{\alpha}
$$

where $C_{1}$ and $C_{2}$ are fitting constants. For networks that follow classical affine deformation, or neo-Hookean behavior, $C_{2}=0$ and $2 C_{1}$ is the shear modulus of the network[36, 47]. For alternative elastic behavior, the Mooney-Rivlin plot provides insight into strain hardening and softening of the material during deformation. Mooney-Rivlin plots of the compressive stressdeformation curves for the water-swollen PEG-co-(PEG-chol) networks are presented in Figure 13. 

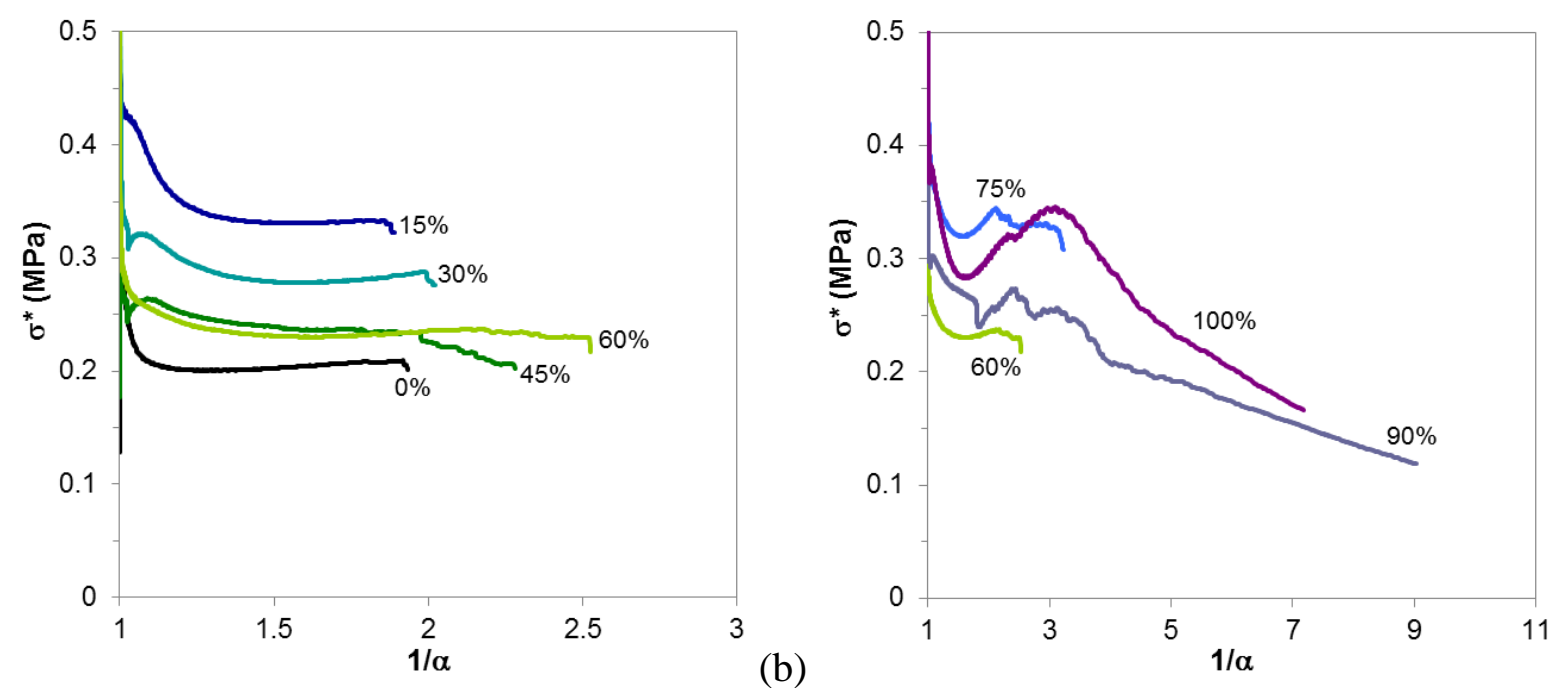

(a)

(b)

Figure 13. Mooney-Rivlin plots of the PEG-co-(PEG-chol) hydrogels undergoing uniaxial compression. (a) 0-60\% PEG-chol networks with cholesterol collapsed into the crosslinks exhibit classical affine behavior. (b) 60-100\% PEG-chol networks with lamellar-like selfassembled cholesterol domains show fluctuating behavior that increases with PEG-chol concentration.

PEG-co-(PEG-chol) hydrogels with ordered crosslinks (0-60\% PEG-chol, 0-2\% chol by swollen weight) exhibit classical affine behavior as the reduced stress is nearly constant and independent of the amount of deformation. Fitting the curves to the Mooney-Rivlin equation (Table 2) confirmed that $2 C_{2}$ is nearly zero for all PEG-chol concentrations below $75 \%$, and so the $2 C_{1}$ fitting constant is equivalent to the shear modulus, $G$, of each network. The elastic modulus, $E$, can be determined through its relation to the shear modulus, $E \cong 3 G$, which is a good approximation for elastomers[48]. Table 2 demonstrates that networks with cholesterol aggregated near the crosslink junctions are mechanically stronger than the standard PEG hydrogel. In fact, incorporating only $15 \%$ PEG-chol into the network nearly doubles the elastic modulus. Below $60 \%$ PEG-chol, the swelling ratios of the resulting hydrogels were equivalent, so the elevated elastic modulus is attributed solely to cholesterol aggregation at the hydrophobic crosslinks. Greater incorporation of PEG-chol increases the contribution of physical crosslinking to the mechanical strength. However, in contrast to the swelling behavior, the strength 
enhancement provided by more hydrophobic cholesterol in the network does not compensate for the strength lost by its replacement of elastically effective PEG-diacrylate chains. Thus, the elastic modulus decreases with increasing addition of PEG-chol to the network. The counter effect of this strength loss is a moderate increase in ductility, as observed in the stressdeformation curves in Figure 12. While the covalent, high functionality PEG crosslinks provide mechanical strength, they are also a source of brittleness due to their inability to make conformational adjustments during deformation. Conversely, physically aggregated cholesterol molecules are not bound into place; their ability to rearrange configurations creates a more flexible network that can redistribute and balance tension during deformation, thereby enduring further strain.

Table 2. Mooney-Rivlin fitting constants and modulus values for PEG-co-(PEG-chol) hydrogel networks under uniaxial compression.

\begin{tabular}{cccc}
\hline $\begin{array}{c}\text { PEG-chol\% } \\
\text { (wt/total solids wt) }\end{array}$ & $2 \mathrm{C}_{1}$ & $2 \mathrm{C}_{2}$ & $\begin{array}{c}\text { Elastic modulus, } E \\
(\mathrm{MPa})\end{array}$ \\
\hline 0 & $0.20 \pm 0.01$ & $-0.001 \pm 0.007$ & $0.60 \pm 0.04$ \\
15 & $0.36 \pm 0.02$ & $-0.020 \pm 0.015$ & $1.08 \pm 0.07$ \\
30 & $0.30 \pm 0.02$ & $-0.009 \pm 0.009$ & $0.90 \pm 0.06$ \\
45 & $0.24 \pm 0.03$ & $-0.004 \pm 0.010$ & $0.71 \pm 0.09$ \\
60 & $0.23 \pm 0.02$ & $0.001 \pm 0.011$ & $0.68 \pm 0.06$ \\
75 & $0.31 \pm 0.03$ & $0.004 \pm 0.008$ & $0.92 \pm 0.09$ \\
\hline
\end{tabular}

The flexibility of aggregated cholesterol upon deformation was especially prominent in the PEG-co-(PEG-chol) networks containing lamellar-like domains (75-100\% PEG-chol, 3.3$6.3 \%$ chol by swollen weight) where physically self-assembled cholesterol dominates the mechanical properties. The large increase in hydrogel ductility despite the relatively low mass percentage of cholesterol in the swollen networks indicates the huge impact of molecular 
redistribution on mechanical behavior. Besides substantially increasing the gel breaking point, the lamellar-like cholesterol domains produce non-linear behavior of the reduced stress, $\sigma^{*}$, over increasing deformation, as shown by the Mooney-Rivlin plots. The fluctuations indicate stress softening and hardening during deformation, and they develop gradually with increased lamellar domain presence in the network. The 75\% PEG-chol networks still display affine-like behavior, and the calculated elastic modulus (Table 2) indicates that the lamellar-like cholesterol ordering creates stronger physical crosslinks than aggregation at the chemical crosslink junctions (35\% increase in $E$ from $60 \%$ PEG-chol). At 100\% PEG-chol, lamellar-like domains are present throughout the network, and an established minimum and maximum are identified in the Mooney-Rivlin plot, indicating a pattern of stress softening at low deformations, stress hardening at intermediate deformations, and more softening at high deformation.

A better understanding of the fluctuating stress softening and hardening of the $90 \%$ and 100\% PEG-chol hydrogel networks can be gathered from the compressive mechanical behavior of liquid crystal polymer networks. These networks, which contain liquid crystals in either the backbone or as side chains, can be crosslinked at high temperatures while the liquid crystals are isotropically distributed and then cooled to form polydomains of layered liquid crystals surrounded by polymer chains[49-51]. The process is analogous to crosslinking the PEG-chol networks in organic solvent and transferring them to aqueous solvent where they self-assemble into many small lamellar-like domains. Crosslinking before transition to the liquid crystal phase generates flexibility for the liquid crystals to rearrange into new formations upon macroscopic deformation[49]. Modeling of a liquid crystal polymer undergoing uniaxial compression shows transitions from an isotropic to para-nematic to nematic phase[52]. A Mooney-Rivlin plot shows initial strain hardening as the isotropic phase is compressed followed by a peak as the network 
transitions to a para-nematic phase and begins to soften from molecular alignment of the liquid crystals. The para-nematic to nematic phase transition causes a discontinuity in the slope, although stress softening is exhibited in both states. While the model in this case described alignment of individual liquid crystal molecules, similar concepts apply to the alignment of randomly oriented liquid crystal polydomains under mechanical deformation. This was seen experimentally by Shandryuk and coworkers during uniaxial compression of a polydomain sidechain liquid crystal polymer network containing smectic layers stabilized by hydrogen bonding[50]. After an initial linear increase in the stress-strain curve, a significant leveling off occurred at a threshold strain around 20\%. X-ray scattering of the polymer films revealed a randomly distributed polydomain structure before compression and a well-ordered monodomain structure after the threshold strain. Finkelmann and colleagues have used biaxial deformation to create a liquid crystalline elastomer with a uniform smectic conformation[53]. Upon uniaxial compression, the monodomain network also exhibited threshold behavior related to either tilting of the liquid crystals or shearing of the stacked layers[54].

Knowing how liquid crystal polydomains respond to mechanical stress allows interpretation of the three stages of behavior detailed in the 100\% PEG-chol Mooney-Rivlin plot. At low strain, the network exhibits softening behavior that was displayed to some extent by all the PEG-co-(PEG-chol) hydrogels. This suggests that some initial softening may occur due to water weeping out of the hydrogel. Deformation of the amorphous regions in the PEG-chol network brings the polydomains of self-assembled, meso-ordered cholesterol closer together, and this leads to strain hardening at intermediate deformations while the orientations of the lamellarlike domains are randomly distributed. Upon further deformation, the cholesterol domains are expected to act analogously to the liquid crystal polymer networks such that they slowly align to 
form a monodomain of cholesterol lamellae, a process described by the second phase of stress softening. In many samples, a slight change in slope is noticeable during the high strain softening phase. As observed in the transition from a paranematic to nematic phase, the slope change could indicate the point of monodomain formation. Continued softening after this point could be caused by either tilting of the stacked cholesterol layers or shearing of the layers atop one another.

\section{Conclusion}

We have demonstrated a method for incorporating cholesterol into a hydrogel network using organic solvent synthesis followed by solvent exchange. This method reduces limitations caused by cholesterol insolubility or phase separation, and thus increases flexibility in the chemical composition of the network. Tethering cholesterol to the network via a PEG-linker promotes aggregation into hydrophobic clusters that act as physical crosslinks. At lower concentrations $(<12 \%$ cholesterol among total solids), cholesterol aggregation around the PEG network crosslinks improves mechanical properties by enhancing the elastic modulus or decreasing brittleness without altering other properties such as hydrogel swelling. More significantly, self-assembly at higher concentrations (15-20\% cholesterol among total solids) develops lamellar-like meso-ordered polydomains within the swollen network, a direct result of the tethered cholesterol structure. These ordered domains, similar to those of a side-chain liquid crystal network, dramatically alter the hydrogel response to deformation and greatly improve the network fracture strength.

\section{Acknowledgments}

This research was supported by a Stanford Graduate Fellowship and by the Center on Polymer Interfaces and Macromolecular Assemblies, an NSF Materials Research Science and 
Engineering Center. Portions of this research were carried out at the Stanford Synchrotron

Radiation Lightsource (SSRL), a national user facility operated by Stanford University on behalf

of the U.S. Department of Energy, Office of Basic Energy Sciences. The authors thank John

Pople at SSRL for assistance with SAXS measurements on beamline 1-4 and Anne Martel and

Thomas Weiss at SSRL for assistance with measurements on beamline 4-2.

\section{Appendix A. Supplementary data}

Additional figures (S01-S04) can be found in the supplementary data.

\section{References}

1. Chen J, Park H, and Park K. Journal of Biomedical Materials Research 1999;44(1):53-62.

2. $\quad$ Okumura Y and Ito K. Advanced Materials 2001;13(7):485-487.

3. Pourjavadi A, Ayyari M, and Amini-Fazl MS. European Polymer Journal 2008;44(4):1209-1216.

4. Darvishi Z, Kabiri K, Zohuriaan-Mehr MJ, and Morsali A. Journal of Applied Polymer Science 2011;120(6):3453-3459.

5. Mahdavinia GR, Pourjavadi A, Hosseinzadeh H, and Zohuriaan MJ. European Polymer Journal 2004;40(7):1399-1407.

6. Muhr AH and Blanshard JMV. Polymer 1982;23(7):1012-1026.

7. Peppas NA and Reinhart CT. Journal of Membrane Science 1983;15(3):275-287.

8. Reinhart CT and Peppas NA. Journal of Membrane Science 1984;18(0):227-239.

9. Tan H, Ramirez CM, Miljkovic N, Li H, Rubin JP, and Marra KG. Biomaterials 2009;30(36):6844-6853.

10. Chung Y-M, Simmons KL, Gutowska A, and Jeong B. Biomacromolecules 2002;3(3):511-516.

11. Jeong B, Windisch CF, Park MJ, Sohn YS, Gutowska A, and Char K. The Journal of Physical Chemistry B 2003;107(37):10032-10039.

12. Jeong B and Bae YH. Nature 1997;388(6645):860-862.

13. Wu L and Ding J. Biomaterials 2004;25(27):5821-5830.

14. Kamath KR and Park K. Advanced Drug Delivery Reviews 1993;11(1-2):59-84.

15. Myung D, Koh W, Ko J, Hu Y, Carrasco M, Noolandi J, Ta CN, and Frank CW. Polymer 2007;48(18):5376-5387.

16. Weng L, Gouldstone A, Wu Y, and Chen W. Biomaterials 2008;29(14):2153-2163.

17. Rakovsky A, Marbach D, Lotan N, and Lanir Y. Journal of Applied Polymer Science 2009;112(1):390-401.

18. Cui J, Lackey MA, Tew GN, and Crosby AJ. Macromolecules 2012;45(15):6104-6110.

19. Haraguchi K and Takehisa T. Advanced Materials 2002;14(16):1120-1124.

20. Sun JY, Zhao X, Illeperuma WRK, Chaudhuri O, Oh KH, Mooney DJ, Vlassak JJ, and Suo Z. Nature 2012;489(7414):133-136. 
21. Cui J, Lackey MA, Madkour AE, Saffer EM, Griffin DM, Bhatia SR, Crosby AJ, and Tew GN. Biomacromolecules 2012;13(3):584-588.

22. Gong JP, Katsuyama Y, Kurokawa T, and Osada Y. Advanced Materials 2003;15(14):1155-1158.

23. Gong JP. Soft Matter 2010;6(12):2583-2590.

24. Nakajima T, Furukawa H, Tanaka Y, Kurokawa T, and Gong JP. Journal of Polymer Science Part B: Polymer Physics 2011;49(17):1246-1254.

25. Sakai T, Matsunaga T, Yamamoto Y, Ito C, Yoshida R, Suzuki S, Sasaki N, Shibayama M, and Chung U-i. Macromolecules 2008;41(14):5379-5384.

26. Shibayama M. Soft Matter 2012;8(31):8030-8038.

27. Waters DJ, Engberg K, Parke-Houben R, Hartmann L, Ta CN, Toney MF, and Frank CW. Macromolecules 2010;43(16):6861-6870.

28. Matsunaga T, Sakai T, Akagi Y, Chung U-i, and Shibayama M. Macromolecules 2009;42(4):1344-1351.

29. Matsunaga T, Sakai T, Akagi Y, Chung U-i, and Shibayama M. Macromolecules 2009;42(16):6245-6252.

30. Qiu Y and Park K. Advanced Drug Delivery Reviews 2012;64:49-60.

31. Waters DJ, Engberg K, Parke-Houben R, Ta CN, Jackson AJ, Toney MF, and Frank CW. Macromolecules 2011;44(14):5776-5787.

32. Elbert DL and Hubbell JA. Biomacromolecules 2001;2(2):430-441.

33. Myung D, Farooqui N, Waters D, Schaber S, Koh W, Carrasco M, Noolandi J, Frank CW, and Ta CN. Current Eye Research 2008;33:29-43.

34. Cruise GM, Scharp DS, and Hubbell JA. Biomaterials 1998;19(14):1287-1294.

35. Ozdemir C and Guner A. European Polymer Journal 2007;43(7):3068-3093.

36. Rubinstein M and Colby RH. Polymer Physics. Oxford, New York: Oxford University Press Inc., 2003.

37. Mark JE and Flory PJ. Journal of the American Chemical Society 1965;87(7):1415-1423.

38. Alexandridis P, Olsson U, and Lindman B. Langmuir 1998;14(10):2627-2638.

39. Holmqvist P, Alexandridis P, and Lindman B. The Journal of Physical Chemistry B 1998;102(7):1149-1158.

40. Shieh HS, Hoard LG, and Nordman CE. Nature 1977;267(5608):287-289.

41. Gupta RK and Suresh KA. European Physical Journal E -- Soft Matter 2004;14:35-42.

42. Holmberg K, Jonsson B, Kronberg B, and Lindman B. Surfactants and Polymers in Aqueous Solution. West Sussex, England: John Wiley \& Sons Ltd, 2003.

43. Claesson M, Engberg K, Frank CW, and Andersson M. Soft Matter 2012;8(31):81498156.

44. $\quad$ Xu P and Mark JE. Polymer 1992;33(9):1843-1848.

45. Mooney M. Journal of Applied Physics 1940;11(9):582-592.

46. Rivlin RS and Saunders DW. Philosophical Transactions of the Royal Society of London. Series A, Mathematical and Physical Sciences 1951;243(865):251-288.

47. Akagi Y, Katashima T, Katsumoto Y, Fujii K, Matsunaga T, Chung U-i, Shibayama M, and Sakai T. Macromolecules 2011;44(14):5817-5821.

48. Sperling LH. Introduction to Physical Polymer Science. Hoboken, New Jersey: John Wiley \& Sons, Inc., 2006.

49. Biggins JS, Warner M, and Bhattacharya K. Journal of the Mechanics and Physics of Solids 2012;60(4):573-590. 
50. Shandryuk GA, Kuptsov SA, Shatalova AM, Plate NA, and Talroze RV. Macromolecules 2003;36(9):3417-3423.

51. Ortiz C, K. Ober C, and Kramer EJ. Polymer 1998;39(16):3713-3718.

52. Bladon P and Warner M. Macromolecules 1993;26(5):1078-1085.

53. Nishikawa E, Yamamoto J, Yokoyama H, and Finkelmann H. Macromolecular Rapid Communications 2004;25(5):611-617.

54. Kramer D and Finkelmann H. Macromolecular Rapid Communications 2011;32(19):1539-1545. 


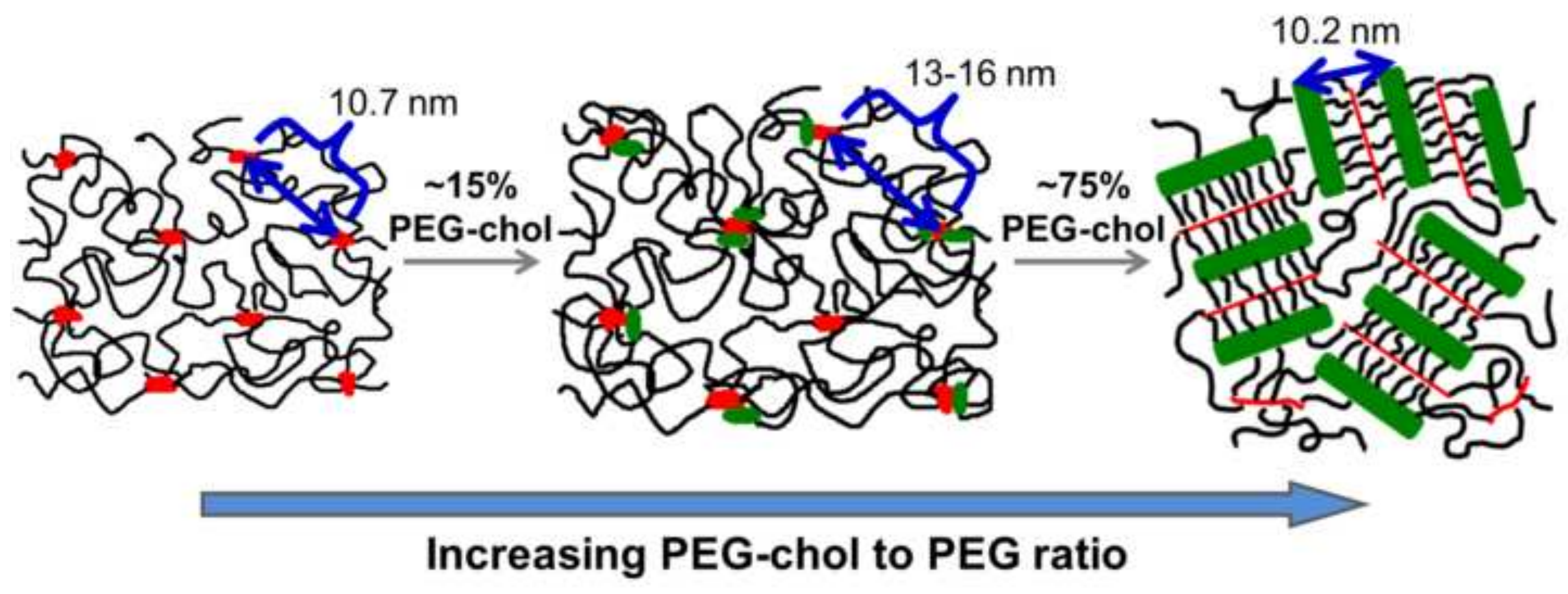

